\title{
Expression of Gastrin-Releasing Peptide Receptor in Breast Cancer and Its Association with Pathologic, Biologic, and Clinical Parameters: A Study of 1,432 Primary Tumors
}

\author{
Clément Morgat ${ }^{1-3}$, Gaétan MacGrogan ${ }^{4,5}$, Véronique Brouste ${ }^{6}$, Valérie Vélasco ${ }^{4,5}$, Nicolas Sévenet $^{5,7}$, Hervé Bonnefoi $^{5,8}$, \\ Philippe Fernandez ${ }^{1-3}$, Marc Debled ${ }^{8}$, and Elif Hindié ${ }^{1-3}$ \\ ${ }^{I}$ Nuclear Medicine Department, University Hospital of Bordeaux, F-33076 Bordeaux, France; ${ }^{2}$ University Bordeaux, INCIA, UMR \\ 5287, F-33400 Talence, France; ${ }^{3}$ CNRS, INCIA, UMR 5287, F-33400 Talence, France; ${ }^{4}$ Surgical Pathology Unit, Department of \\ BioPathology, Institut Bergonié, F-33076 Bordeaux, France; ${ }^{5}$ INSERM, ACTION U1218, F-33076 Bordeaux, France; ${ }^{6}$ Clinical and \\ Epidemiological Research Unit, Institut Bergonié, F-33076 Bordeaux, France; ${ }^{7}$ Oncogenetics Unit, Department of BioPathology, \\ Institut Bergonié, F-33076 Bordeaux, France; and ${ }^{8}$ Department of Medical Oncology, Institut Bergonié, F-33076 Bordeaux, France
}

A growing body of evidence suggests that gastrin-releasing peptide receptor (GRPR) might be a valuable target in breast cancer. To understand which patients can be potential candidates for GRPRbased imaging or targeted therapy, we screened invasive breast cancers by immunohistochemistry for the presence and intensity of GRPR expression. Methods: We explored a tissue microarray of 1,432 primary breast tumors from patients who underwent surgery between 2000 and 2005 at Institut Bergonié, without prior neoadjuvant treatment. We studied associations between GRPR expression and clinical, pathologic, and biologic parameters. The association between GRPR expression and distant metastasis-free interval was also examined. Results: GRPR overexpression was found in $75.8 \%$ of the 1,432 tumors and was most strongly associated with estrogen receptor (ER) positivity (GRPR was high in $83.2 \%$ of ER-positive and $12 \%$ of ER-negative tumors; $P<0.00001$ ). When molecular subtypes of breast cancer were considered, GRPR was overexpressed in $86.2 \%$ of luminal A-like tumors, $70.5 \%$ of luminal B-like human epidermal growth factor receptor 2 (HER2)-negative tumors, 82.8\% of luminal B-like HER2-positive tumors, $21.3 \%$ of HER2enriched tumors, and $7.8 \%$ of triple-negative tumors. Importantly, when breast tumors overexpressed GRPR, high GRPR expression was also found in metastatic lymph nodes in $94.6 \%$ of cases. Primary tumors with high GRPR expression were associated with lower risk of distant metastases at follow-up in univariate analysis (Log-rank $P=0.0084$ ) but not in multivariate analysis. Hence, the prognostic impact of GRPR was lost when examined within specific molecular subtypes. Conclusion: Because GRPR is overexpressed in a high percentage of ER-positive tumors, GRPR targeting offers wide perspectives for imaging and treatment in patients with ER-positive breast cancer, using recently developed radiolabeled GRPR ligands.

Key Words: GRPR; breast cancer; imaging; ${ }^{68} \mathrm{Ga}$; ${ }^{177} \mathrm{Lu}$; PRRT

J Nucl Med 2017; 58:1401-1407

DOI: 10.2967/jnumed.116.188011

Received Dec. 6, 2016; revision accepted Feb. 17, 2017.

For correspondence or reprints contact: Clément Morgat, Nuclear Medicine Department, University Hospital of Bordeaux, 33000 Bordeaux, France.

E-mail: clement.morgat@chu-bordeaux.fr

Published online Mar. 9, 2017.

COPYRIGHT (C 2017 by the Society of Nuclear Medicine and Molecular Imaging.
$\mathbf{T}$ he overexpression in tumors of receptors for some regulatory peptides offers the possibility of targeting these receptors for imaging or treatment. A well-known example is the targeting of somatostatin receptors in neuroendocrine tumors. PET with radiolabeled somatostatin analogs (e.g. ${ }^{68} \mathrm{Ga}$-DOTATOC, ${ }^{68} \mathrm{Ga}$-DOTATATE) is the most exquisite means of imaging these tumors (1), whereas targeted therapy using similar analogs labeled with $\beta$-emitting radionuclides (e.g., ${ }^{90}$ Y-DOTATOC or ${ }^{177} \mathrm{Lu}$-DOTATATE) offers excellent results in metastatic patients (2). Receptors for other peptides are overexpressed in many tumors, raising hopes for similar achievements (3).

The gastrin-releasing peptide receptor (GRPR) is a G-proteincoupled receptor of the family of bombesin receptors (3-5). GRPR overexpression was found in small cell lung cancer (6) and then in many tumors, including prostate, breast, colon, and pancreatic cancer; head and neck squamous cell tumors; neuroblastomas; and gliomas $(4,5)$.

After early reports on the presence of GRPR in some breast cancer cell lines (7), Halmos et al. studied the binding of the bombesin analog ${ }^{125} \mathrm{I}_{-} \mathrm{Tyr}^{4}$-BN on cell membranes from $100 \mathrm{hu}-$ man breast carcinomas; $33 \%$ of the tumors expressed bombesin/ GRPR at levels greater than $10 \mathrm{fmol} / \mathrm{mg}$ membrane protein and there was a positive correlation between the binding capacity of bombesin and estrogen receptor (ER) levels (8). Using autoradiography, Gugger and Reubi found GRPR to be expressed in 32 of $52(62 \%)$ invasive breast carcinomas. GRPR was also expressed, although heterogeneously, in normal breast tissue (9). Dalm et al. studied frozen sections from 50 breast tumors with autoradiography using the GRPR agonist ${ }^{111}$ In-AMBA; GRPR was expressed in $96 \%$ of specimens (10). In another study, they found that high GRPR messenger mRNA (mRNA) levels were more frequent in samples with positivity for estrogen receptor mRNA ESR1, or progesterone receptor (PR) mRNA, with negativity for ERBB2 status, and in samples with favorable genomic grade (11). These studies show that GRPR is a relevant target in breast cancer, although the rate of GRPR positivity varies from one study to another.

Importantly, radiolabeled GRPR antagonists for PET imaging recently offered interesting results in prostate and breast cancer patients (12-14). ${ }^{68} \mathrm{Ga}$-SB3 showed good targeting in 4 of 6 patients with metastatic breast cancer (13). In another study, ${ }^{68} \mathrm{Ga}-\mathrm{RM} 2$ visualized 13 of 18 breast tumors, with high intensity compared 
with normal breast tissue, and detected metastatic lesions (14). GRPR antagonists have also now been labeled with therapy radionuclides (e.g., $\left.{ }^{177} \mathrm{Lu}-\mathrm{RM} 2,{ }^{177} \mathrm{Lu}-\mathrm{NeoBOMB} 1\right)(15,16)$.

Selection of appropriate candidates for clinical trials aiming at GRPR imaging or therapy requires precise knowledge of which tumors might overexpress the receptors. Using immunohistochemistry (IHC), we aimed at elucidating GRPR expression in the complex landscape of breast cancer.

\section{MATERIALS AND METHODS}

\section{Tissue Microarray}

Samples from 1,432 invasive breast cancers from patients having received initial surgery at Institut Bergonié between 2000 and 2005 were included in this study. Quadruplicate cores, $0.6 \mathrm{~mm}$ in diameter each, of Hollande-fixed tumor samples were used for the construction of the tissue microarray (TMA). No patient had received neoadjuvant hormone therapy or chemotherapy. After surgery, adjuvant radiotherapy or systemic therapy (hormonal, chemotherapy) was offered according to prevailing guidelines; trastuzumab as adjuvant treatment was not used during this period. Clinical follow-up data had been prospectively entered into a database and were available for this study.

All IHC and fluorescence in situ hybridization (FISH) analyses were performed on the TMA. Human epidermal growth factor receptor 2 (HER2) status was determined according to published recommendations (17). Molecular subtypes of breast cancers were derived from IHC markers, based on ER status, PR status, Ki-67-labeling index, and HER2 status, according to St Gallen consensus (18) and Maisonneuve classification (19).

Molecular subtypes were defined as follows: luminal A-like (ER $\geq$ $1 \%$; HER 2 score $0,1+$, or $2+$ with no HER2 amplification by FISH analysis and $\mathrm{Ki}-67<14 \%$ or $\mathrm{Ki}-67$ ranging from $14 \%$ to $19 \%$; and $\mathrm{PR} \geq 20 \%$ ); luminal B-like HER2 - (ER $\geq 1 \%$; HER2 score $0,1+$, or $2+$ with no HER2 amplification by FISH analysis and Ki-67 $\geq$ $20 \%$ or Ki-67 14\% to $19 \%$; and PR $<20 \%$ ); luminal B-like HER2+ (ER $\geq 1 \%$; HER2 score $3+$ or $2+$ with HER2 amplification by FISH analysis); HER2-enriched (HER2 score $3+$ or $2+$ with HER2 amplification by FISH analysis, $\mathrm{ER}=0 \%$ and $\mathrm{PR}=0 \%$ ); and triple-negative $(\mathrm{ER}=0 \%$; PR $=0 \%$; HER2 score $0,1+$, or $2+$ with no HER2 amplification by FISH analysis).

As regards androgen receptor (AR), tumors were considered AR+ if $10 \%$ or more of the nuclei were stained, independent of intensity.

Characteristics of our series are presented in Supplemental Table 1 (supplemental materials are available at http://jnm.snmjournals.org).

The Ethics committee of Institut Bergonié waived requirement for informed consent for the present retrospective analysis.

\section{GRPR IHC}

Rules for G-protein-coupled receptor IHC studies in human tissues were strictly adhered to (20). Briefly, our validation steps of the IHC assay included: Western blotting of breast cancer cell line lysates, immunochemistry of these cell lines fixed and embedded with the same material as that used for construction of TMA, subcellular localization of the immunostaining, and identification of positive and negative tumors within a dedicated TMA and validation of the signal recorded in IHC by Western blotting of corresponding frozen tumors from patient samples. Then, the TMA of 1,432 tumors was analyzed.

For IHC staining on TMA, 4- $\mu$ m-thick paraffin-embedded tissue sections were mounted on slides. The IHC procedure was conducted using the Ventana Benchmark-ultra automated staining system and according to the manufacturer's recommendations. Primary antibodies and procedures are detailed in Supplemental Table 2. IHC results for GRPR were assessed under light microscope by a senior pathologist and expressed as an immunoreactive score (IRS) (21) that took into account staining intensity ( 0 for no staining, 1 for weak staining, 2 for moderate staining, and 3 for strong staining) and the percentage of stained tumor cells ( 0 for no positive cells, 1 for $\leq 10 \%$ positive cells, 2 for $11 \%-50 \%$ positive cells, 3 for $51 \%-80 \%$ positive cells, and 4 for $>80 \%$ of positive cells). The final IRS score (staining intensity $\times$ percentage of positive cells) thus ranged from 0 to 12 . For the purpose of our study, no GRPR expression referred to IRS 0-1, weak GRPR expression referred to IRS 2-3, moderate GRPR expression meant IRS 4-8, and strong GRPR expression meant IRS 9-12.

\section{Statistical Analysis}

For association with other parameters, GRPR data were dichotomized into 2 groups: low GRPR (absent/weak GRPR expression) and high GRPR (moderate/strong GRPR expression). We studied associations between GRPR expression and the various parameters displayed in Supplemental Table 1. Differences between categorized variables were assessed with the $\chi^{2}$ test or Fischer exact test. All $P$ values were 2 -sided, and a $P$ value of less than 0.05 was considered statistically significant. Factors that are predictive of high GRPR expression (moderate/ strong) were investigated by univariate and multivariate analysis using a logistic regression. Odds ratios and their $95 \%$ confidence intervals $(95 \%$ CIs) were reported.

The median follow-up was calculated by the reverse Kaplan-Meier method. Probabilities of overall survival (OS) and distant metastasisfree interval (DMFI) were calculated with the Kaplan-Meier method. The log-rank test was used for comparison between GRPR groups. For OS, all causes of death were considered as events. For DMFI, all distant metastatic events were considered and patients with no metastases were censored at the date of last news. Multivariate analyses for DMFI were calculated with a Cox model. Variables significant at the 0.05 level in the univariate analysis were introduced in the multivariate model, which used a stepwise descending maximum likelihood

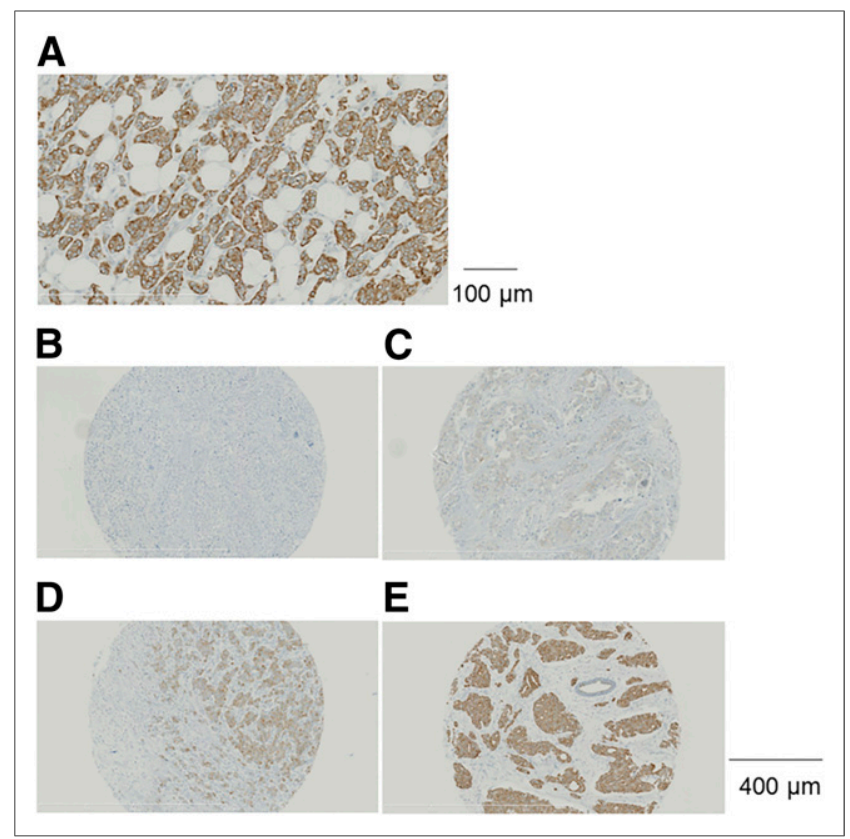

FIGURE 1. (A) Membrane and cytoplasmic localization of GRPR immunostaining in a breast cancer specimen (magnification, $\times 20$ ). (B-E) Representative samples of breast cancer specimens showing the 4 categories of GRPR immunostaining results: no GRPR expression (IRS score 0 or 1) (B), weak GRPR expression (IRS score 2 or 3 ) (C), moderate GRPR expression (IRS score 4-8) (D), and strong GRPR expression (IRS score 9-12) (E) (magnification, $\times 10$ ). 
method. Hazard ratios (HRs) and their 95\% CIs were reported. For validity of the Cox model, proportionality was tested with the residuals test. For lymph node status, patients who did not receive lymph node dissection $(\mathrm{Nx})$ were kept in the prognostic model as a separate category.

Statistical analyses were performed using the SAS software (version 9.3; SAS Institute, Inc.).

\section{Lymph Node Metastases (Ancillary Study)}

In addition to our investigation of the TMA of primary tumors, we studied 37 lymph node metastases from 37 patients (1 lymph node per patient) whose primary breast tumors were positive for GRPR. This ancillary study aimed at verifying that GRPR expression is maintained in metastatic tissue.

\section{RESULTS}

\section{Validation Steps for GRPR IHC}

Analysis of various human breast cancer cell lines (MCF-7, ZR75.1, SKBR3, MB-453, MDA-MB-468, and T47-D) revealed a good correlation between GRPR signal recorded with Western blot and that seen with immunocytochemistry (Supplemental Table 3). Western blots showed a single band at the expected GRPR molecular weight (Supplemental Fig. 1).
Analysis of tumoral tissue from 34 patients showed good correlation of GRPR signals at IHC on Holland-fixed paraffin-embedded material and on Western blot analyses of corresponding fresh frozen tissue (Supplemental Fig. 1B).

\section{GRPR Expression in 1,432 Breast Cancers and Its Association with Pathologic and Biologic Parameters}

GRPR exhibited a predominantly intracytoplasmic expression in tumor cells and with concomitant immunoreactivity of the cell membrane. This distribution fits the established criteria on IHC for G-protein-coupled receptors (20). Tumor-associated stromal tissue showed either no or weak expression of GRPR.

Among 1,432 invasive breast cancers, GRPR expression was absent in $145(10.1 \%$ ) tumors (IRS score $=0$ or 1 ), weak in 202 $(14.1 \%)$ tumors (IRS score $=2$ or 3 ), moderate in $512(35.8 \%)$ tumors (IRS score $=4-8$ ), and strong in 573 (40.0\%) tumors (IRS score $=9-12$ ). Representative IHC samples are shown in Figure 1.

When results were dichotomized into 2 groups to study associations with other parameters, 347 tumors $(24.2 \%)$ were GRPR-low (absent/ weak) and 1,085 tumors (75.8\%) were GRPR-high (moderate/strong).

Associations between GRPR expression (high vs. low) and various pathologic and biologic parameters are summarized in Table 1. GRPR-high was more frequent in tumors from women

TABLE 1

Distribution of Low and High GRPR Expression Within Different Categories of Breast Cancers

\begin{tabular}{|c|c|c|c|c|c|}
\hline \multirow[b]{2}{*}{ Characteristic } & \multirow[b]{2}{*}{ Category } & \multirow[b]{2}{*}{ No. of patients } & \multicolumn{2}{|c|}{ GRPR expression } & \multirow[b]{2}{*}{$P$} \\
\hline & & & Low \% & High \% & \\
\hline \multirow[t]{2}{*}{ Age (y) } & $\leq 40 y$ & 85 & 45.9 & 54.1 & $<0.00001$ \\
\hline & $>40 y$ & 1,347 & 22.9 & 77.1 & \\
\hline \multirow[t]{2}{*}{ Histology* } & IDC & 1,221 & 25.4 & 74.6 & 0.09 \\
\hline & ILC & 161 & 19.3 & 80.7 & \\
\hline \multirow[t]{2}{*}{ Pathologic size (mm) } & $<20$ & 961 & 20.9 & 79.1 & 0.00003 \\
\hline & $\geq 20$ & 471 & 31.0 & 69.0 & \\
\hline \multirow[t]{3}{*}{ SBR grade* } & 1 & 358 & 10.6 & 89.4 & $<0.00001^{\dagger}$ \\
\hline & II & 661 & 16.9 & 83.1 & \\
\hline & III & 397 & 48.6 & 51.4 & \\
\hline \multirow[t]{2}{*}{ Ki-67 } & $<20 \%$ & 1,115 & 17.0 & 83.0 & $<0.00001$ \\
\hline & $\geq 20 \%$ & 317 & 49.8 & 50.2 & \\
\hline \multirow[t]{2}{*}{ Lymph node status* } & $\mathrm{N}_{0}$ & 848 & 23.3 & 76.7 & 0.03 \\
\hline & $\mathrm{N}^{+}$ & 472 & 28.8 & 71.2 & \\
\hline \multirow[t]{2}{*}{ ER } & $0 \%$ & 150 & 88.0 & 12.0 & $<0.00001$ \\
\hline & $\geq 1 \%$ & 1,282 & 16.8 & 83.2 & \\
\hline \multirow[t]{2}{*}{ PR } & $0 \%$ & 266 & 60.9 & 39.1 & $<0.00001$ \\
\hline & $\geq 1 \%$ & 1,166 & 15.9 & 84.1 & \\
\hline \multirow[t]{2}{*}{ AR } & $<10 \%$ & 184 & 63.0 & 37.0 & $<0.00001$ \\
\hline & $\geq 10 \%$ & 1,248 & 18.5 & 81.5 & \\
\hline \multirow[t]{2}{*}{ HER2 overexpression } & No & 1,286 & 22.8 & 77.2 & 0.0001 \\
\hline & Yes & 146 & 37.0 & 63.0 & \\
\hline
\end{tabular}

${ }^{*}$ Patients with other histologic subtypes $(n=50)$ are excluded from statistical analysis. Number of patients with missing information regarding items: SBR grade, $n=16$; lymph node status $\left(\mathrm{N}_{\mathrm{x}}\right), n=112$. Statistical analysis based on $\chi^{2}$ test.

${ }^{\dagger} P$ value was calculated for SBR grade I + II vs. III.

IDC = invasive ductal carcinoma; ILC = invasive lobular carcinoma. 
older than $40 \mathrm{y}(77.1 \%$ vs. $54.1 \% ; P<0.00001)$ and in tumors less than $20 \mathrm{~mm}(79.1 \%$ vs. $69 \%$; $P=0.00003)$, whereas no difference was observed between invasive ductal carcinomas and invasive lobular carcinomas (74.6\% vs. $80.7 \% ; P=0.09)$. GRPR-high was more frequent in SBR (Scarff-Bloom-Richardson) grades I/II than in grade-III tumors $(85.3 \%$ vs. $51.4 \% ; P<0.00001)$ and in tumors with $\mathrm{Ki}-67<20 \%$ than in tumors with Ki-67 $\geq 20 \%(83 \%$ vs. $50.2 \% ; P<0.00001)$. GRPR-high was slightly more frequent in tumors from patients without nodal involvement (76.7\% vs. $71.2 \%$; $P=0.03$ ). There was a striking difference in the rate of GRPR-high between ER-positive and ER-negative tumors (83.2\% vs. $12 \% ; P<$ 0.00001) (Table 1). GRPR-high was also more frequent in PR-positive tumors $(84.1 \%$ vs. $39.1 \% ; P<0.00001)$ and in AR-positive tumors ( $81.5 \%$ vs. $37 \%$; $P<0.00001)$. GRPR was high in $77.2 \%$ of HER2negative and in $63 \%$ of HER2-positive tumors $(P=0.0001)$.

Table 2 shows results of univariate and multivariate analyses of factors predictive for high GRPR expression. In multivariate analysis, ER positivity was the most important predictor (odds ratio, 11.998; 95\% CI, 5.980-24.075; $P<0.0001)$.

\section{GRPR Expression in ER-Positive Tumors}

High GRPR expression in ER-positive tumors makes this group of particular interest as regards GRPR targeting for imaging or therapy. Among the 1,282 ER-positive tumors, GRPR was highly expressed in $83.2 \%$ of tumors overall, with only small variations between different categories (Table 3). GRPR-high is somewhat more frequent in women older than $40 \mathrm{y}(83.9 \%$ vs. $70.8 \% ; P=$ 0.006), in tumors less than $20 \mathrm{~mm}(85.1 \%$ vs. $79.2 \% ; P=0.009)$, in tumors with lower SBR grade (I and II vs. III, 86.8\% vs.70.4\%; $P<0.00001)$ or with Ki-67 less than $20 \%$ (86.7\% vs. $70 \% ; P<$ $0.00001)$, in PR-positive tumors $(84.1 \%$ vs. $74.1 \% ; P=0.006)$, and in AR-positive tumors ( $84.5 \%$ vs. $67 \% ; P=0.00002)$. There was no influence of HER2 status, histologic subtype (invasive ductal carcinoma vs. invasive lobular carcinoma), or nodal status $\left(\mathrm{N}^{+}\right.$vs. $\mathrm{N}_{0}$ ) (Table 3$)$.

There was no linearity between the percentage of ER-positive cells and the probability of GRPR overexpression (Pearson linear correlation coefficient $=0.403$ ). However, the 19 tumors with only 1\%-9\% ER-positive cells had lower rates of GRPR overexpression (55.6\% vs. $83.6 \% ; P=0.005)$.

TABLE 2

Univariate and Multivariate Analysis of Predictors of High GRPR Expression

\begin{tabular}{|c|c|c|c|c|c|c|}
\hline \multirow[b]{2}{*}{ Characteristic } & \multirow[b]{2}{*}{ Category } & \multirow[b]{2}{*}{ No. of patients } & \multicolumn{2}{|c|}{ Univariate analysis } & \multicolumn{2}{|c|}{ Multivariate analysis } \\
\hline & & & $P$ & Odds ratio & $P$ & Odds ratio \\
\hline \multirow[t]{3}{*}{ Age (y) } & $<40$ & 85 & $<0.0001$ & 1 & $\begin{array}{r}\text { Nonsignificant } \\
\text { (not retained) }\end{array}$ & \\
\hline & $41-50$ & 287 & $<0.0001$ & 2.897 (1.742-4.816) & & \\
\hline & $>50$ & 1,060 & $<0.0001$ & $2.851(1.818-4.472)$ & & \\
\hline \multirow[t]{3}{*}{ Histology } & IDC & 1,221 & 0.0389 & 1 & Not retained & \\
\hline & ILC & 161 & 0.0910 & $1.427(0.945-2.155)$ & & \\
\hline & Other & 43 & 0.0479 & $2.586(1.009-6.628)$ & & \\
\hline \multirow[t]{2}{*}{ Pathologic size $(\mathrm{mm})$} & $\geq 20$ & 471 & $<0.0001$ & 1 & Not retained & \\
\hline & $<20$ & 961 & & $1.699[1.324 ; 2.180]$ & & \\
\hline \multirow[t]{3}{*}{ SBR grade } & Grade III & 397 & $<0.0001$ & 1 & 0.0005 & 1 \\
\hline & Grade II & 661 & $<0.0001$ & 4.637 (3.495-6.154) & 0.0023 & $1.831(1.242-2.699)$ \\
\hline & Grade I & 358 & $<0.0001$ & 7.965 (5.395-11.761) & 0.0002 & 2.617 (1.591-4.303) \\
\hline \multirow[t]{2}{*}{$\mathrm{Ki}-67$} & $\geq 20 \%$ & 317 & $<0.0001$ & 1 & 0.0196 & 1 \\
\hline & $<20 \%$ & 1,115 & & 4.869 (3.716-6.378) & & $1.584(1.076-2.330)$ \\
\hline \multirow[t]{3}{*}{ Lymph node status } & $\mathrm{N}^{+}$ & 472 & 0.0006 & 1 & 0.0350 & 1 \\
\hline & $\mathrm{N}_{0}$ & 848 & 0.0289 & $1.329(1.030-1.715)$ & 0.3117 & $1.173(0.861-1.597)$ \\
\hline & Missing & 112 & 0.0003 & $3.082(1.672-5.681)$ & 0.0103 & $2.636(1.257-5.526)$ \\
\hline \multirow[t]{2}{*}{ ER } & $0 \%$ & 150 & $<0.0001$ & 1 & $<0.0001$ & 1 \\
\hline & $\geq 1 \%$ & 1,282 & & 36.394 (21.772-60.837) & & $11.998(5.980-24.075)$ \\
\hline \multirow[t]{2}{*}{ PR } & $0 \%$ & 266 & $<0.0001$ & 1 & 0.0429 & 1 \\
\hline & $\geq 1 \%$ & 1,166 & & $8.259(6.167-11.061)$ & & $1.652(1.016-2.686)$ \\
\hline \multirow[t]{2}{*}{ AR } & $<10 \%$ & 184 & $<0.0001$ & 1 & 0.0027 & 1 \\
\hline & $\geq 10 \%$ & 1,248 & & 7.509 (5.389-10.462) & & $2.011(1.275-3.173)$ \\
\hline \multirow{2}{*}{$\begin{array}{l}\text { HER2 } \\
\text { overexpression }\end{array}$} & Yes & 146 & 0.0002 & 1 & 0.0038 & 1 \\
\hline & No & 1,286 & & $1.989(1.387-2.852)$ & & $0.461(0.273-0.779)$ \\
\hline
\end{tabular}

IDC = invasive ductal carcinoma; ILC = invasive lobular carcinoma.

Statistical analysis based on Cox model; $P<0.05$ was considered significant. Data in parentheses are $95 \%$ Cls. 
TABLE 3

Distribution of Low and High GRPR Expression Within Different Categories of ER-Positive Tumors

\begin{tabular}{|c|c|c|c|c|c|}
\hline \multirow[b]{2}{*}{ Characteristic } & \multirow[b]{2}{*}{ Category } & \multirow[b]{2}{*}{ No. of patients } & \multicolumn{2}{|c|}{ GRPR expression } & \multirow[b]{2}{*}{$P$} \\
\hline & & & Low \% & High \% & \\
\hline \multirow[t]{2}{*}{ Age (y) } & $\leq 40$ & 65 & 29.2 & 70.8 & 0.006 \\
\hline & $>40$ & 1,217 & 16.1 & 83.9 & \\
\hline \multirow[t]{2}{*}{ Histology* } & IDC & 1,080 & 17.1 & 82.9 & 0.70 \\
\hline & ILC & 158 & 18.4 & 81.6 & \\
\hline \multirow[t]{2}{*}{ Pathologic size $(\mathrm{mm})$} & $<20$ & 883 & 14.9 & 85.1 & 0.009 \\
\hline & $\geq 20$ & 399 & 20.8 & 79.2 & \\
\hline \multirow[t]{3}{*}{ SBR grade* } & I & 356 & 10.1 & 89.9 & $<0.00001^{\dagger}$ \\
\hline & ॥ & 643 & 14.9 & 85.1 & \\
\hline & III & 267 & 29.6 & 70.4 & \\
\hline \multirow[t]{2}{*}{$\mathrm{Ki}-67$} & $<20 \%$ & 1,072 & 13.3 & 86.7 & $<0.00001$ \\
\hline & $\geq 20 \%$ & 210 & 30.0 & 70.0 & \\
\hline \multirow[t]{2}{*}{ Lymph node status ${ }^{\star}$} & $\mathrm{N}_{0}$ & 762 & 16.0 & 84.0 & 0.052 \\
\hline & $\mathrm{N}^{+}$ & 414 & 20.5 & 79.5 & \\
\hline \multirow[t]{2}{*}{ Estrogen receptor } & $1 \%-9 \%$ & 18 & 44.4 & 55.6 & $0.005^{\ddagger}$ \\
\hline & $\geq 10 \%$ & 1,264 & 16.4 & 83.6 & \\
\hline \multirow[t]{2}{*}{ PR } & $0 \%$ & 116 & 25.9 & 74.1 & 0.006 \\
\hline & $\geq 1 \%$ & 1,166 & 15.9 & 84.1 & \\
\hline \multirow[t]{2}{*}{ AR } & $<10 \%$ & 91 & 33.0 & 67.0 & 0.00002 \\
\hline & $\geq 10 \%$ & 1,191 & 15.5 & 84.5 & \\
\hline \multirow[t]{2}{*}{ HER2 overexpression } & No & 1,183 & 16.7 & 83.3 & 0.91 \\
\hline & Yes & 99 & 17.2 & 82.8 & \\
\hline
\end{tabular}

${ }^{*}$ Patients with other histologic subtypes $(n=44)$ are excluded from statistical analysis. Number of patients with missing information regarding items: SBR grade, $n=16$; lymph node status $\left(\mathrm{N}_{\mathrm{x}}\right), n=106$.

${ }^{\dagger} P$ value was calculated for SBR grade I + II vs. III.

${ }^{\ddagger}$ Statistical analysis based on $\chi^{2}$ or Fischer test.

IDC = invasive ductal carcinoma; ILC = invasive lobular carcinoma.

\section{GRPR Expression in ER-Negative Tumors}

When ER-negative tumors were considered together $(n=150)$, high GRPR expression was found in only $12 \%$. GRPR-high was more frequent in HER2-positive tumors $(21.3 \%$ vs. $7.8 \% ; P=0.018)$ or in AR-positive tumors $(19.3 \%$ vs. $7.5 \% ; P=0.03)$. No other associations were found (Supplemental Table 4).

\section{GRPR Expression in Different Molecular Subtypes of Breast Cancer}

GRPR-high was found in $86.2 \%$ of luminal A-like tumors, in $70.5 \%$ of luminal B-like HER2-negative tumors, and in $82.8 \%$ of luminal B-like HER2-positive tumors. Among ER-negative subtypes, only $21.3 \%$ of HER2-enriched cases and $7.8 \%$ of triplenegative breast cancer cases were GRPR-high (Table 4).

\section{GRPR Expression and Outcomes}

Median follow-up was 122 mo. Distant metastases occurred in 153 patients. There were 198 deaths overall.

Figure 2 shows Kaplan-Meier curves for OS and DMFI according to GRPR status. There was no difference in OS (Log-rank $P=$ 0.1056). Less distant metastases occurred in the GRPR-high group (Log-rank $P=0.0084$ ). Ten-year DMFI was $89.9 \%$ in the GRPRhigh group and $85.1 \%$ in the GRPR-low group.
Among the 1,282 ER-positive cases (Fig. 2C), there also was less distant metastases in the GRPR-high group (log-rank $P=$ 0.0355). However, when examined within molecular subgroups, there was no association between GRPR expression and DMFI (luminal-A, $P=0.0874$; luminal-B, $P=0.9394$ ) (Figs. 2D and 2E). Also, in a multivariate analysis of factors associated with DMFI in ER-positive cases, only tumor size $(>2 \mathrm{~cm}, \mathrm{HR}=2.2)$,

TABLE 4

Distribution of Low and High GRPR Expression Within Different Molecular Subtype of Breast Cancers

\begin{tabular}{|c|c|c|c|c|}
\hline \multirow[b]{2}{*}{ Subtype } & \multirow{2}{*}{$\begin{array}{c}\text { No. of } \\
\text { patients }\end{array}$} & \multicolumn{2}{|c|}{$\begin{array}{c}\text { GRPR } \\
\text { expression }\end{array}$} & \multirow[b]{2}{*}{$P$} \\
\hline & & Low \% & High \% & \\
\hline Luminal A & 963 & 13.8 & 86.2 & $<0.00001$ \\
\hline Luminal B HER2- & 220 & 29.5 & 70.5 & \\
\hline Luminal B HER2+ & 99 & 17.2 & 82.8 & \\
\hline HER2-enriched & 47 & 78.7 & 21.3 & \\
\hline Triple-negative & 103 & 92.2 & 7.8 & \\
\hline
\end{tabular}




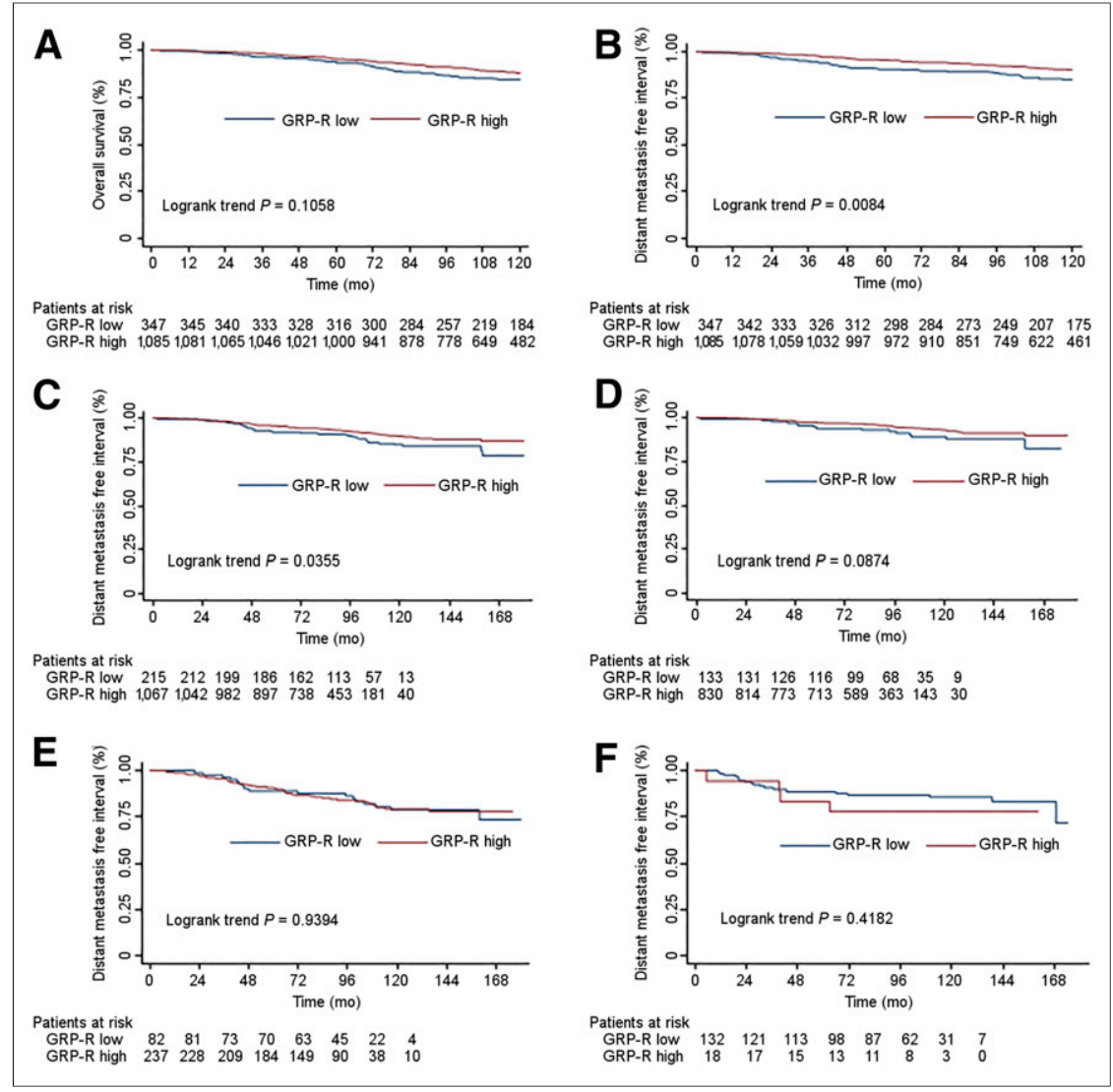

FIGURE 2. (A) Association of GRPR expression in primary tumors with OS in 1,432 patients. (B) DMFI in 1,432 patients according to GRPR expression in primary breast tumor. (C) DMFI in ERpositive tumors as a function of GRPR expression. (D) DMFI in luminal-A tumors as a function of GRPR expression. (E) DMFI in luminal-B tumors as a function of GRPR expression. (F) Distant MFI in ER-negative tumors as a function of GRPR expression.

tumor grade (grade II vs. grade I HR = 3.0; grade III vs. grade I $\mathrm{HR}=2.6)$ and positive node status $(\mathrm{HR}=2.2)$ were significant prognostic factors (Supplemental Table 5).

In ER-negative tumors, there was no association between GRPR expression and DMFI $(P=0.42)$ (Fig. 2F).

\section{GRPR Expression in Lymph Node Metastases from Patients with GRPR-Positive Breast Tumor (Ancillary Study)}

Among the 37 studied lymph nodes metastases, GRPR was absent in $1(2.7 \%)$, weak in $1(2.7 \%)$, moderate in $23(62.2 \%)$, and strong in $12(32.4 \%)$. When results were dichotomized, as performed for primary tumors, 2 (5.4\%) lymph nodes metastases were GRPRlow and $35(94.6 \%)$ were GRPR-high. Staining distribution was mostly homogeneous.

\section{DISCUSSION}

The overexpression of GRPR in breast cancer raises hopes for imaging $(13,14)$, for radioligand therapy $(3,15,16,22)$, or for selective delivery of chemotherapy or nanoparticles with diverse designs (23). Comprehending the full potential of GRPR targeting requires precise knowledge of which tumors might overexpress the receptors.

In the present study of 1,432 breast cancers, there was a striking association between GRPR expression and ER status. GRPR overexpression at IHC was found in $83.2 \%$ of ER-positive tumors and in only $12 \%$ of ER-negative tumors $(P<0.00001)$ (Table 1$)$.
These results agree with earlier findings $(8,10,11)$. To further understand associations with GRPR, we performed a multivariate analysis taking into account multiple clinicopathologic and biologic parameters. ER positivity was the most important predictor of high-GRPR expression (OR: $\sim 12$; $P<0.0001$ ) (Table 2).

Within the ER-positive subgroup, there were only small variations in GRPR overexpression (according to tumor size, SBR grade, Ki-67 index, PR status, or AR status); the probability of GRPR overexpression would exceed $70 \%$ in most subsets (Table 3). Trials to assess the correlation between GRPR expression on IHC and uptake on GRPR PET imaging will be useful. As regards tumors with only $1 \%-9 \%$ ERpositive cells, representing about $1.5 \%$ of ER-positive tumors, GRPR overexpression was found in only $55.6 \%$ of cases. In GRPR imaging trials, this subgroup may need to be analyzed separately. When considering molecular subtypes of ER-positive breast cancer, GRPR overexpression was somewhat more frequent in luminal A-like tumors $(86.2 \%)$ than luminal B-like HER2negative tumors $(70.5 \%)$ and luminal B-like HER2-positive tumors $(82.8 \%)$. Given the association between GRPR overexpression and ER positivity, we are currently investigating with IHC the impact of hormonal neoadjuvant therapy on GRPR expression.

The prognostic value of GRPR probability results from its association with ER status. Its prognostic impact is lost when examined within specific molecular subgroups (Fig. 2) or in multivariate analysis (Supplemental Table 5).

An important finding is that GRPR expression is conserved in nodal metastases. We examined lymph nodes from 37 patients with GRPR-positive breast tumors. In 35 cases (94.6\%), lymph nodes metastases were GRPR-high. Staining was mostly homogeneous. These results confirm previous findings from Gugger and Reubi (9) and recent genomic data on GRPR mRNA expression in lymph nodes and distant lesions from Dalm et al. (24).

Pilot studies of imaging with radiolabeled GRPR antagonists are quite encouraging $(13,14) .{ }^{68} \mathrm{Ga}-\mathrm{SB} 3$ showed good targeting in 4 of 6 unselected patients with metastatic breast cancer (13). In another study, ${ }^{68} \mathrm{Ga}$-RM2 visualized 13 of 18 primary tumors and identified metastatic sites (internal mammary nodes, bone lesions). When only ER-positive tumors were considered, ${ }^{68} \mathrm{Ga}-\mathrm{RM} 2 \mathrm{PET}$ identified 13 of 14 tumors (14). These results suggest a potential role for GRPR PET imaging that might be complementary to that of ${ }^{18} \mathrm{~F}$-FDG in ER-positive breast cancer patients, because ${ }^{18} \mathrm{~F}-\mathrm{FDG}$ uptake can be limited in low-grade tumors and in lobular carcinoma (25-27). $16 \alpha-{ }^{18}$ F-fluoro-17 $\beta$-estradiol is a useful tracer because it allows the assessment of ER expression and prediction of the outcome of hormonal treatment (28). The use of GRPR imaging to monitor endocrine therapy also deserves investigation (29).

In light of the presented results, we are planning GRPR imaging trials in ER-positive breast cancer patients. We will examine sensitivity 
in staging patients who have either locally advanced disease or de novo diagnosis of metastatic breast cancer. Comparison will be made with ${ }^{18} \mathrm{~F}$-FDG. We are also planning to study the role of GRPR imaging in assessing response to hormonal treatment in the neoadjuvant setting and in metastatic disease in comparison with $16 \alpha-{ }^{18}$ F-fluoro- $17 \beta$-estradiol.

The specific advantage of GRPR imaging over other tracers for breast cancer is that it offers unique perspectives for targeted radionuclide therapy. When metastases show high uptake on PET imaging with ${ }^{68} \mathrm{Ga}$-labeled GRPR ligands, this means that the same ligands labeled with $\beta$-emitters (e.g., ${ }^{177} \mathrm{Lu},{ }^{90} \mathrm{Y},{ }^{161} \mathrm{~Tb}$ ) might be envisaged for targeted therapy (22). Pharmacologic side effects encountered with agonist analogs of GRPR (abdominal cramps, emesis) (30) are avoided when using antagonists (15). Some ${ }^{177} \mathrm{Lu}$-labeled GRPR antagonists have been tested in preclinical studies, with excellent tumor targeting, and are expected to enter clinical trials $(15,16)$. These preclinical data point to the pancreas as the dose-limiting organ regarding the density of GRPR expression. Importantly, the pharmacokinetics of ${ }^{177} \mathrm{Lu}-\mathrm{RM} 2$ show much faster clearance from the pancreas than tumors (15). It has also been shown with ${ }^{177} \mathrm{Lu}-\mathrm{NeoBOMB} 1$ that the tumor-topancreas uptake ratios can be highly improved by careful selection of the amount of injected peptide (16). Thus, GRPR-targeted therapy using radiolabeled antagonists opens new perspectives in ERpositive patients with distant metastases who failed prior therapies.

One limitation to our study on breast cancer TMA is that we could not assess GRPR in nonneoplastic breast tissue compartments. Recent results of PET/CT imaging with the GRPR antagonist ${ }^{68} \mathrm{Ga}-\mathrm{RM} 2$ showed only low to moderate uptake in normal breast (14).

\section{CONCLUSION}

Our findings on a large tumor bank of primary operable breast cancer show that GRPR is overexpressed in $83 \%$ of ER-positive tumors. When the primary tumor had high GRPR expression, this overexpression was also found in lymph node metastases in $94.6 \%$ of cases. Clinical trials in ER-positive breast cancer will assess the value of GRPR targeting for imaging or therapy, a promising avenue.

\section{DISCLOSURE}

This work was funded by the French Investment for the Future program within LabEx TRAIL ANR-10-LABX-57. No other potential conflict of interest relevant to this article was reported.

\section{ACKNOWLEDGMENTS}

We thank Colette Riès, Lionnelle Belanga, and Catherine Savona-Baron for their precious assistance.

\section{REFERENCES}

1. Deroose CM, Hindié E, Kebebew E, et al. Molecular imaging of gastroenteropancreatic neuroendocrine tumors: current status and future directions. $\mathrm{J} \mathrm{Nucl}$ Med. 2016;57:1949-1956.

2. Strosberg J, El-Haddad G, Wolin E, et al. Phase 3 trial of ${ }^{177} \mathrm{Lu}$-Dotatate for midgut neuroendocrine tumors. N Engl J Med. 2017;376:125-135.

3. Morgat C, Mishra AK, Varshney R, Allard M, Fernandez P, Hindié E. Targeting neuropeptide receptors for cancer imaging and therapy: perspectives with bombesin, neurotensin, and neuropeptide-Y receptors. J Nucl Med. 2014;55:16501657.

4. Hohla F, Schally AV. Targeting gastrin releasing peptide receptors: new options for the therapy and diagnosis of cancer. Cell Cycle. 2010;9:1738-1741.

5. Ramos-Álvarez I, Moreno P, Mantey SA, et al. Insights into bombesin receptors and ligands: highlighting recent advances. Peptides. 2015;72:128-144.
6. Moody TW, Carney DN, Cuttitta F, Quattrocchi K, Minna JD. High affinity receptors for bombesin/GRP-like peptides on human small cell lung cancer. Life Sci. 1985;37:105-113.

7. Giacchetti S, Gauville C, de Cremoux P, et al. Characterization, in some human breast cancer cell lines, of gastrin-releasing peptide-like receptors which are absent in normal breast epithelial cells. Int J Cancer. 1990;46:293-298.

8. Halmos G, Wittliff JL, Schally AV. Characterization of bombesin/gastrin releasing peptide receptors in human breast cancer and their relationship to steroid receptor expression. Cancer Res. 1995;55:280-287.

9. Gugger M, Reubi JC. Gastrin-releasing peptide receptors in non-neoplastic and neoplastic human breast. Am J Pathol. 1999;155:2067-2076.

10. Dalm SU, Martens JW, Sieuwerts AM, et al. In-vitro and in-vivo application of radiolabeled gastrin releasing peptide receptor ligands in breast cancer. $J \mathrm{Nucl}$ Med. 2015;56:752-757.

11. Dalm SU, Sieuwerts AM, Look MP, et al. Clinical relevance of targeting the gastrin releasing peptide receptor, somatostatin receptor 2 or chemokine c-x-c motif receptor 4 in breast cancer for imaging and therapy. J Nucl Med. 2015;56:1487-1493.

12. Kähkönen E, Jambor I, Kemppainen J, et al. In vivo imaging of prostate cancer using $\left[{ }^{68} \mathrm{Ga}\right]$-labeled bombesin analog BAY86-7548. Clin Cancer Res. 2013;19:54345443.

13. Maina T, Bergsma H, Kulkarni HR, et al. Preclinical and first clinical experience with the gastrin-releasing peptide receptor-antagonist $\left[{ }^{68} \mathrm{Ga}\right] \mathrm{SB} 3$ and PET/CT. Eur J Nucl Med Mol Imaging. 2016;43:964-973.

14. Stoykow C, Erbes T, Maecke H, et al. Gastrin-releasing peptide receptor imaging in breast cancer using the receptor antagonist ${ }^{68} \mathrm{Ga}-\mathrm{RM} 2$ and PET. Theranostics. 2016;6:1641-1650.

15. Dumont RA, Tamma M, Braun F, et al. Targeted radiotherapy of prostate cancer with a gastrin-releasing peptide receptor antagonist is effective as monotherapy and in combination with rapamycin. J Nucl Med. 2013;54:762-769.

16. Dalm SU, Bakker IL, de Blois E, et al. ${ }^{68} \mathrm{Ga} /{ }^{177} \mathrm{Lu}-\mathrm{NeoBOMB} 1$, a novel radiolabeled GRPR antagonist for theranostic use in oncology. J Nucl Med. 2017;58:293-299.

17. Wolff AC, Hammond ME, Hicks DG, et al. Recommendations for human epidermal growth factor receptor 2 testing in breast cancer: American Society of Clinical Oncology/College of American Pathologists clinical practice guideline update. J Clin Oncol. 2013;31:3997-4013.

18. Goldhirsch A, Winer EP, Coates AS, et al. Personalizing the treatment of women with early breast cancer: highlights of the St Gallen international expert consensus on the primary therapy of early breast cancer 2013. Ann Oncol. 2013;24:22062223.

19. Maisonneuve P, Disalvatore D, Rotmensz N, et al. Proposed new clinicopathological surrogate definitions of luminal A and luminal B (HER2-negative) intrinsic breast cancer subtypes. Breast Cancer Res. 2014;16:R65.

20. Reubi JC. Strict rules are needed for validation of G-protein-coupled receptor immunohistochemical studies in human tissues. Endocrine. 2014;47:659-661.

21. Remmele W, Stegner H. Recommendation for uniform definition of an immunoreactive score (IRS) for the immunohistochemical estrogen receptor detection (ER-ICA) in breast cancer tissue [in German]. Pathologe. 1987;8:138-140.

22. Hindié E, Zanotti-Fregonara P, Quinto M, Morgat C, Champion C. Dose deposits from ${ }^{90} \mathrm{Y},{ }^{177} \mathrm{Lu},{ }^{111} \mathrm{In}$, and ${ }^{161} \mathrm{~Tb}$ in micrometastases of various sizes: implications for radiopharmaceutical therapy. J Nucl Med. 2016;57:759-764.

23. Wang C, Sun X, Wang K, Wang Y, Yang F, Wang H. Breast cancer targeted chemotherapy based on doxorubicin-loaded bombesin peptide modified nanocarriers. Drug Deliv. 2016;23:2697-2702.

24. Dalm SU, Schrijver WA, Sieuwerts AM, et al. Prospects of targeting the gastrin releasing peptide receptor and somatostatin receptor 2 for nuclear imaging and therapy in metastatic breast cancer. PLoS One. 2017;12:e0170536.

25. Groheux D, Giacchetti S, Moretti JL, et al. Correlation of high ${ }^{18}$ F-FDG uptake to clinical, pathological and biological prognostic factors in breast cancer. Eur J Nucl Med Mol Imaging. 2011;38:426-435.

26. Groheux D, Hindie E, Delord M, et al. Prognostic impact of ${ }^{18}$ FDG-PET-CT findings in clinical stage III and IIB breast cancer. $J$ Natl Cancer Inst. 2012;104:1879-1887.

27. Hogan MP, Goldman DA, Dashevsky B, et al. Comparison of ${ }^{18}$ F-FDG PET/CT for systemic staging of newly diagnosed invasive lobular carcinoma versus invasive ductal carcinoma. J Nucl Med. 2015;56:1674-1680.

28. Liao GJ, Clark AS, Schubert EK, Mankoff DA. ${ }^{18}$ F-fluoroestradiol PET: current status and potential future clinical applications. J Nucl Med. 2016;57:12691275 .

29. Prignon A, Nataf V, Provost C, et al. ${ }^{68} \mathrm{Ga}-\mathrm{AMBA}$ and ${ }^{18} \mathrm{~F}-\mathrm{FDG}$ for preclinical PET imaging of breast cancer: effect of tamoxifen treatment on tracer uptake by tumor. Nucl Med Biol. 2015;42:92-98.

30. Bodei L, Ferrari M, Nunn A. ${ }^{177}$ Lu-AMBA bombesin analogue for hormone refractory prostate cancer patients: a phase I escalation study with single-cycle administrations [abstract]. Eur J Nucl Med Mol Imaging. 2007;34(suppl 2):S221. 